\title{
How to approach a patient with hand tremor
}

\author{
Dharshana Sirisena \\ Neurology Unit Teaching Hospital Anuradhapura
}

\section{Introduction}

Tremor is an oscillatory, rhythmic involuntary movement of a body part and is the commonest movement disorder ${ }^{1-4}$. Though this can involve any part of the body, hands are the commonest. Diagnosis is based on history, good observation of the tremor, guided by relevant clinical examination aided by investigations when indicated. This article reviews the tremor classification, associated syndromes and evaluation of a patient.

\section{Classification}

Tremors are classified in different ways as it has numerous etiologies and lack of a practical etiological or physiological classification. Therefore the clinical classification still accepted widely as the gold standard ${ }^{1}$.

Clinically tremors are mainly classified into rest and action. Latter is further classified into postural, isometric and kinetic tremor where intension tremor is included under kinetic tremor. Rest tremor occurs when the body part is not voluntarily activated and completely supported against gravity (hands rest on the laps).This usually augments with mental stress(counting backwards) and relieves with goal directed movements.
Action tremor is any tremor that is produced by voluntary contraction of muscles. Postural tremor is present while voluntarily maintaining a position against gravity as in extending arms in front of the body. Isometric tremor occurs as a result of muscle contraction against rigid stationary object (squeezing the examiners fingers).

Kinetic tremor occurs during any voluntary movement and is simple when occurs during nontarget directed movements (flexion /extension, pronation / supination at wrist) and intension when is target directed as in finger-nose test. Kinetic tremor could be task specific when it appears or augments during specific tasks as in writing. ${ }^{1-6}$

Phenomenology of tremor helps in determining the cause of the tremor, but further clinical evaluation is necessary for determination of different tremor syndromes. Characteristics of different tremors are summarized in table 1. 


\begin{tabular}{|c|c|c|c|c|}
\hline $\begin{array}{l}\text { Type of } \\
\text { tremor }\end{array}$ & Frequency $(\mathrm{Hz})$ & amplitude & occurrence & Associations \\
\hline Rest & $\begin{array}{l}\text { Low to } \\
\text { medium } \\
(3-6)\end{array}$ & $\begin{array}{l}\text { High } \\
\text { (reduced } \\
\text { with target } \\
\text { directed } \\
\text { movements) }\end{array}$ & $\begin{array}{l}\text { Lib supported } \\
\text { against gravity } \\
\text { with muscles } \\
\text { not activated }\end{array}$ & Parkinson's Disease , drug induced \\
\hline Action & - & - & $\begin{array}{l}\text { Voluntary } \\
\text { muscle } \\
\text { contraction }\end{array}$ & \\
\hline Postural & $\begin{array}{l}\text { Medium to } \\
\text { high } \\
(4-12)\end{array}$ & $\begin{array}{l}\text { low, } \\
\text { increases } \\
\text { with } \\
\text { voluntary } \\
\text { movements }\end{array}$ & $\begin{array}{l}\text { Limbs } \\
\text { maintains } \\
\text { position } \\
\text { against gravity }\end{array}$ & $\begin{array}{l}\text { Physiological, essential } \\
\text { tremor,thyrotoxicosis, hypoglycemia, al } \\
\text { cohol withdrawal and drugs }\end{array}$ \\
\hline $\begin{array}{l}\text { Simple } \\
\text { kinetic }\end{array}$ & Variable(3-10) & $\begin{array}{l}\text { Does not } \\
\text { change with } \\
\text { target- } \\
\text { directed } \\
\text { movements. }\end{array}$ & $\begin{array}{l}\text { Simple } \\
\text { movements of } \\
\text { the limb }\end{array}$ & $\begin{array}{l}\text { Pronation-supintion or flexion } \\
\text { extension of wrist }\end{array}$ \\
\hline Intention & Low $(<5)$ & $\begin{array}{l}\text { Increases } \\
\text { with target } \\
\text { directed } \\
\text { movements }\end{array}$ & $\begin{array}{l}\text { Target } \\
\text { directed } \\
\text { movements. }\end{array}$ & $\begin{array}{l}\text { Cerebellar lesions (multiple sclerosis, } \\
\text { stroke, tumours) } \\
\text { drugs, chronic alcoholism }\end{array}$ \\
\hline Isometric & Medium & variable & $\begin{array}{l}\text { Muscle } \\
\text { contraction } \\
\text { against } \\
\text { stationary } \\
\text { object }\end{array}$ & Holding an object in the hand \\
\hline $\begin{array}{l}\text { Task } \\
\text { specific }\end{array}$ & Variable $(4-10)$ & variable & specific tasks & $\begin{array}{l}\text { Primary writing tremor, musician's } \\
\text { tremor }\end{array}$ \\
\hline
\end{tabular}

Table 1. Characteristics of different tremors

\section{Physiologic tremor}

Every normal person exhibits a physiologic tremor, a benign high frequency, low amplitude, postural tremor. This may well be demonstrated when holding a piece of paper on the outstretched hand. Exagerated physiologic tremor is visible and occurs in the absence of neurological disease ${ }^{1}$. When encountered associations such as thyrotoxicosis, hypoglycemia, alcohol withdrawal, use of certain drugs should be excluded.Propranolol may be used for symptomatic relief but alleviation of the cause usually relieves the tremor ${ }^{1,2}$.

\section{Essential Tremor (ET)}

ET is the commonest adult onset movement disorder with a prevalence of 4.1-39.2 cases per 1000 and increasing to 50.5 in elderly, above $60 \mathrm{yrs}^{8}$. It is usually bilateral, largely symmetrical postural or kinetic involving hands and forearms. Other features that help in diagnosis are gradual and yet progressive with increase in amplitude over the time and spread to involve neck and voice in the absence of abnormal posturing(dystonia), acute reduction of tremor amplitude in response to alcohol and familial inheritance. . $^{1-4 \cdot 9-12}$ 
If tremor is disabling and embarrassing enough to merit treatment, non-selective beta-blocker, and propranolol is the main stay of treatment.Anti convulsant primidone is equally effective ${ }^{11-13}$.In resistant disabling cases Deep Brain Stimulation, a mode of surgical treatment is indicated following thorough assessment.

\section{Dystonic Tremor}

This is tremor occurring in the body part affected by dystonia.Dystonic head tremor is classical of this and should be differentiated from tremor associated with dystonia in which tremor occurs in a body part not affected by dystonia. ${ }^{1,13,14}$ Upper limb postural tremor occurring in a cervical dystonia patient is an example that could be difficult to differentiate clinically from ET where electrophysiological studies could be helpful.

Orthostatic tremor is a unique tremor syndrome where sufferers feel unsteadiness during standing causing enormous social embarrassment. Diagnosis should be confirmed by demonstrating a $13-18 \mathrm{~Hz}$ high frequency tremor on electrophysiological studies.

\section{Parkinsonian Tremor}

Parkinson's disease (PD) is relatively less common than ET ${ }^{8}$.About 70000 Australians are suffering from PD (Access Economics).As specific treatment options are available early diagnosis is mandatory. When a patient has any form of pathological tremor together with other diagnostic criteria of PD they are having Pakinsonian tremor syndrome. Tremor may be the presenting feature and when coexistence with one or more typical features of PD diagnosis is most likely.Bradykinasia is a must, whereas rigidity and postural instability are the other main associated features ${ }^{15-17}$.
A pure rest tremor with low frequency $(4-6 \mathrm{~Hz}$ ) or rest and postural/kinetic with same frequency is classical of PD. Postural/kinetic with different frequencies which may or may not be associated with rest tremor are also seen ${ }^{1}$. At times the tremor may be disabling. The tremor can spread to involve the am and even the head and face in advanced disease.

\section{Cerebellar tremor}

A pure or a dominant intension tremor at a frequency of less than $5 \mathrm{~Hz}$ is classical. Tremor should be absent at rest but may be associated with posture. Tremor could either be uni-or bilateral depending on the etiology.Stroke, multiple sclerosis, tumors, drug toxicity are common culprits. It is essential to look into other cerebellar signs as tremor usually occurs in combination in cerebellar lesions ${ }^{18}$.

\section{Holmes' tremor}

This rare slow tremor is a combination of rest and intension tremor. A postural component is also seen in many patients. This is of central origin associated with lesion in the CNS (mid brain, thalamus) ${ }^{19}$

\section{Drug-induced and Toxic tremor (Table 2)}

This considered as drug-induced if occurs after a reasonable time frame following drug ingestion. Toxic tremor occurs after intoxication ${ }^{1}$.

Dug-induced tremors could have the whole spectrum of clinical features of tremor. Common once are enhanced physiological tremor seen with sympathomimetics and anti depressants and classic Parkinson's tremor associated with neuroleptics and dopamine blocking agents ${ }^{1}$. Alcohol withdrawal usually results in enhanced physiological tremor which needs to differentiate from intension tremor 
secondary to cerebellar damage in chronic alcoholism.

Toxic tremors are usually associated with other clinical signs of CNS intoxication such as gaze abnormalities and gait disturbance ${ }^{1}$.

\section{Tremor syndromes in peripheral neuropathy}

Many peripheral neuropathies are associated with tremors which are postural and kinetic predominantly. These peripherally originated tremors are commonly seen with demyelinating neuropathies especially with dysgammaglobulinemias ${ }^{1}$.

\section{Psychogenic tremors}

This may have different clinical presentations. Sudden onsets and remissions with unusual clinical combinations of tremors should raise the suspicion. Disappearance and change in frequency of tremor when co-activating the antagonistic muscles (resistance to passive movements about a joint) in the tremulous limb is a useful clinical sign to demonstrate (coactivation sign). ${ }^{20}$ Distraction reduces the amplitude together with variation of tremor frequency. Past history of somatization and appearance of additional and unrelated neurological signs are additional evidence ${ }^{1}$.

\section{Evaluation of a tremor patient}

Nothing can be substituted for a good history in evaluating a patient with tremor.Onset, relieving and exacerbating factors, family history and recent medications are essentials. The initial assessment should also include functional limitations mainly

\begin{tabular}{|l|l|l|}
\hline Postural & Intension & Rest \\
\hline Alcohol & Alcohol & Metoclopramide \\
Amiadarone & Lithium & $\begin{array}{l}\text { Neuroleptics } \\
\text { Reserpine }\end{array}$ \\
Amphetamines & & \\
Beat adrenergic agonists. & & \\
Caffeine & & \\
Cyclosporine & & \\
Dopamine & & \\
Steroids & & \\
Theopylline & & \\
Thyroid hormones & & \\
Try cyclic antidepressants & & \\
Valproic acid & & \\
\hline
\end{tabular}

Table 2. Common causes of drug induced tremor

activities of daily living, occupation, social and recreational activities.

Good clinical observation is the most important aspect of physical examination, and should aim at demonstrating various aspects of tremor phenomenology described earlier. Arms resting on the laps, extending in front of the body, finger-nose test are some essential maneuvers and holding a cup, drawing a spiral are also useful in diagnosis as well in determining functional limitations.

Physician should be able to describe tremor as to involve body part (arms, head and neck), activation condition (when it is actually present or get worse), frequency (fast or slow) and amplitude (fine or coarse).

When encountered with a rest tremor it is essential to check for associated rigidity and bradykinasia. Both the tremor and rigidity get worse when performing voluntary movements from opposite limb. Gait is an essential part of assement which could demonstrate difficulty in initiation, reduced arm swinging and freezing. If PD is suspected a trial of levodopa is appropriate and referral to a neurologist is important if features are atypical or failure to respond to medication.

In assessing Patients with intension tremor, associated symptoms like imbalance, dizziness, dysarthria and ataxia should be inquired and looked 
into. Stroke result in acute onset symptoms where as multiple sclerosis demonstrate relapsing nature together with visual disturbance and diverse neurological features. Chronic alcoholism is an important cause that needs to exclude with relevant examination and relevant blood tests.

Thyrotoxicosis is a common presentation of postural tremor which needs exclusion by relevant clinical examination (rapid pulse, eye signs, and goiter) and arranging a thyroid profile. Anxiety and panic attacks are commonly associated with postural tremors and relevant features such as palpitation, chest discomfort and feeling of suffocation should be looked especially in young. Medication over use and withdrawal is an important cause that should look in postural tremors. Essential tremors should be suspected once the clinical examination is otherwise normal especially with a positive family history.

\section{References}

1. Deuschl G, Bain P, Brin M.Consensus statement of the Movement Disorder Society on Tremor. Ad Hoc Scientific Committee.Mov Disord 1998; 13(supp|3):2-23.

2. Hallett M.Classification and treatment of tremor.JAMA 1991; 266:1115-17.

3. Findley LJ, Koller WC.Handbook of tremor Disorders. New York.NY: Marcel Dekker.1995.

4. Zesiewicz TA,Hauser RA.Phenomenology and treatment of tremor disorders. Neurol clin 2001; 19:651-80.

5. Charles PD,Esper GJ,Davis TL, Maciunas RJ,Robertson D.Clasification of tremor ; and update on treatment.Am Fam Physician 1999:59:1665-72.

\section{Investigating a tremor patient}

Diagnosis of tremor is usually clinical but can be supported by electrophysiological studies mainly surface electromyography, accelerometers, handwriting tremor analysis. An EMG assessment of the tremor is the most reliable method of diagnosing primary orthostatic tremor. This can also be useful in diagnosing dystonic tremor and assessing the frequency which help in differentiating difficult tremors. Though not routinely used several rating scales are available for assessing various aspects of tremor syndromes. ${ }^{1,21}$

Routine and specific investigations should be ordered depending on the clinical suspicion of etiology of tremor.e.g. thyroid function tests in exaggerated physiological tremor

6. Smaga S.Tremor.Am Fam Physician.2003; 68:1545-52.

7. Brain PG.Findley LJ.Thompson PD.et al.A study on hereditary essential tremor.Brain.1994.

8. Louis ED, Ottman R, Hauser WA. How common is the most common adult movement disorder? Estimates of the prevalence of essential tremor throughout the world.Mov Disord 1998;13:510.

9. Findley LJ.Koller WC.Essential tremor; a review.Neurology.1987; 3737:1194-97.

10. Lou JS, Jankovic J.Essential tremor; clinical correlates in 350 patients. Neurology.1991; 41:234-38

11. Evidente VG.Understanding essential tremor.Differentialdiagnosis and option for treatment.Post grad Med. 2000; 108:13840,143-46. 
12. Jankovik J.Essential tremor: clinical characteristics. Neurology.2000; 54(suppl 4):s21-25.

13. Jedynak CP, Bonnet AM, Agid Y.Tremor and idiopathic dystonia.Mov Disord 1991;6:230236.

14. Jankovik J, Leder S,Warner D.et al.Cervical dystonia:clinical findings and associated movement disorders. Neurology 1991;41:108891.

15. Uitti RJ.Tremor: How to determine if the patient has Parkinson's disease. Geriatrics 1998;53:3036.

16. Hughes AJ, Daniel SE, Blankson $S$ et al.A clinicopathologic study of 100 cases of Parkinson's disease. Arch Neurol 1993; 50:140148.
17. Anouti A, Koller WC.Diagnostic testing in movement disorders. Neurol clin 2000; 14:16982.

18. Fahn S. Cerebellar tremor: clinical aspects.In: Findley LJ, Capildeo R, eds.Movement Disorders; Tremor.London:Macmillan.1984;355-364.

19. Miwa H, Hatori K, Kondo T, et al.Thalamic tremor: case reports and implications of tremor-generating mechanisms. Neurology 1996;46:75-79.

20. Deuschl G, Koster BCHL,Scheidt C.Diagnostic criteria and clinical course of psychogenic tremor.Mov Disord 1998;13:294-302.

21. Fahn S, Tolosa E, Marin C.Clinical rating scale for tremor.In: Jankovic J,Tolsa E eds. Parkinson's Disease and Movement Disorders. Baltimore. MD:Williams \& Wilkins.1993:271-280.

\section{Author}

